\title{
Erratum to: A meta-analysis of the effect of strontium ranelate on the risk of vertebral and non-vertebral fracture in postmenopausal osteoporosis and the interaction with FRAX ${ }^{\circledR}$
}

\author{
J. A. Kanis - H. Johansson • A. Oden • E. V. McCloskey
}

Published online: 31 May 2011

(C) International Osteoporosis Foundation and National Osteoporosis Foundation 2011

\section{Erratum to: Osteoporos Int \\ DOI 10.1007/s00198-010-1474-0}

An error occurred in this publication due to the incorrect computation of the hazard ratio (HR) for the effects of strontium ranelate. Treatment with strontium ranelate was associated with a decrease in the risk of a clinical vertebral fracture compared with placebo $(\mathrm{HR}=0.75 ; 95 \%$ confidence interval $0.62-0.92$ ). In the original publication the HR was given as 0.50 (95\% CI $0.41-0.60)$. The error does not affect the overall interpretation of the data or conclusions but alters the numerical values given in Tables 3, $4,5,6$. The corrected tables are given below.

The online version of the original article can be found at http://dx.doi. org/10.1007/s00198-010-1474-0.

J. A. Kanis $(\bowtie) \cdot$ H. Johansson $\cdot$ A. Oden $\cdot$ E. V. McCloskey WHO Collaborating Centre for Metabolic Bone Diseases,

University of Sheffield Medical School,

Beech Hill Road,

Sheffield S10 2RX South Yorkshire, UK

e-mail: w.j.Pontefract@shef.ac.uk
Table 3 The relationship of incident fracture (fractures/100 patient years) in placebo-treated patients by quartiles of fracture probability

\begin{tabular}{lllll}
\hline Fracture outcome & \multicolumn{4}{l}{ Quartile } \\
\cline { 2 - 5 } & I & II & III & IV \\
\hline Clinical fractures & & & & \\
All clinical osteoporotic fractures & 4.34 & 6.14 & 7.50 & 10.10 \\
All clinical fractures & 4.78 & 6.72 & 8.05 & 10.62 \\
Non-vertebral OP fractures & 2.97 & 3.43 & 5.36 & 5.72 \\
All non-vertebral fractures & 3.38 & 4.01 & 5.88 & 6.24 \\
Hip fracture & 0.33 & 0.62 & 1.32 & 1.82 \\
Vertebral fractures & & & & \\
Morphometric & 4.68 & 5.60 & 6.56 & 9.41 \\
Clinical vertebral fracture & 1.56 & 2.76 & 2.41 & 4.74 \\
\hline
\end{tabular}

Table 4 Overall effects of strontium ranelate compared to placebo according to the fracture outcome selected

\begin{tabular}{llll}
\hline Fracture outcome & HR & $95 \% \mathrm{CI}$ & $p$ \\
\hline Clinical fractures & & & \\
All & 0.82 & $0.73-0.93$ & $=0.0013$ \\
Osteoporotic & 0.80 & $0.71-0.91$ & $<0.001$ \\
Non-vertebral fractures & & & \\
All & 0.87 & $0.75-1.00$ & $=0.053$ \\
Osteoporotic & 0.84 & $0.72-0.98$ & $=0.028$ \\
Hip & 0.95 & $0.70-1.28$ & $>0.30$ \\
Vertebral fractures & & & \\
Clinical & 0.75 & $0.62-0.92$ & $=0.0044$ \\
Morphometric & 0.60 & $0.52-0.69$ & $<0.001$ \\
\hline
\end{tabular}


Table 5 Hazard ratio between treatments (strontium ranelate versus placebo) for all clinical osteoporotic fractures at different values of 10 year probability (\%) of a major osteoporotic fracture calculated with and without BMD

\begin{tabular}{|c|c|c|c|c|c|c|}
\hline \multirow[t]{2}{*}{ Percentile } & \multicolumn{3}{|c|}{ Probability calculated without BMD } & \multicolumn{3}{|c|}{ Probability calculated with BMD } \\
\hline & 10 year probability & HR & $95 \% \mathrm{CI}$ & 10 year probability & HR & $95 \% \mathrm{CI}$ \\
\hline 10th & $9.0 \%$ & 0.77 & $0.68-0.87$ & $11.5 \%$ & 0.70 & $0.58-0.84$ \\
\hline 25 th & $12.6 \%$ & 0.78 & $0.70-0.88$ & $16.0 \%$ & 0.72 & $0.62-0.85$ \\
\hline 50 th & $18.3 \%$ & 0.82 & $0.73-0.91$ & $22.2 \%$ & 0.76 & $0.67-0.87$ \\
\hline 75 th & $26.0 \%$ & 0.86 & $0.74-0.99$ & $30.2 \%$ & 0.82 & $0.82-0.93$ \\
\hline 90th & $33.5 \%$ & 0.90 & $0.74-1.10$ & $39.8 \%$ & 0.88 & $0.88-1.04$ \\
\hline
\end{tabular}

Table 6 Hazard ratio between treatments (strontium ranelate versus placebo) for hip fracture and for clinical vertebral fracture at different percentiles of 10 year probability (\%) of a major osteoporotic fracture calculated with BMD

\begin{tabular}{|c|c|c|c|c|c|c|}
\hline \multirow[t]{2}{*}{ Percentile } & \multicolumn{3}{|l|}{ Hip fracture } & \multicolumn{3}{|c|}{ Clinical vertebral fracture } \\
\hline & 10 year probability & $\mathrm{HR}$ & $95 \% \mathrm{CI}$ & 10 year probability & HR & $95 \% \mathrm{CI}$ \\
\hline 10th & $11.5 \%$ & 1.03 & $0.63-1.69$ & $11.5 \%$ & 0.65 & $0.49-0.88$ \\
\hline 25 th & $16.0 \%$ & 1.01 & $0.66-1.54$ & $16.0 \%$ & 0.68 & $0.53-0.87$ \\
\hline 50th & $22.2 \%$ & 0.98 & $0.70-1.38$ & $22.2 \%$ & 0.71 & $0.57-0.88$ \\
\hline 75th & $30.2 \%$ & 0.95 & $0.70-1.28$ & $30.2 \%$ & 0.76 & $0.62-0.92$ \\
\hline 90th & $39.8 \%$ & 0.90 & $0.62-1.31$ & $39.8 \%$ & 0.81 & $0.64-1.03$ \\
\hline
\end{tabular}

